Editor's Note: These short reviews of a recent paper in the Journal, written exclusively by graduate students or postdoctoral fellows, are intended to mimic the journal clubs that exist in your own departments or institutions. For more information on the format and purpose of the Journal Club, please see http://www.jneurosci.org/misc/ifa_features.shtml.

\title{
Autobiographical Memory Transformation across Consolidation
}

\author{
Ruud M. W. J. Berkers ${ }^{1,2}$ and Marlieke T. R. van Kesteren ${ }^{1,3}$ \\ ${ }^{1}$ Donders Institute for Brain, Cognition, and Behaviour, Radboud University Nijmegen, 6500 HB Nijmegen, The Netherlands, and Departments of \\ ${ }^{2}$ Cognitive Neuroscience and ${ }^{3}$ Anatomy, Radboud University Nijmegen Medical Centre, 6500 HB Nijmegen, The Netherlands \\ Review of Bonnici et al.
}

One of the greatest mysteries of the brain is how it can recall particular events vividly, sometimes after many decades, whereas the bulk of day-to-day experiences are forgotten. This question has sparked a vigorous search for the memory engram, i.e., the mnemonic representations in the brain. Seminal work by Lashley (1950) suggested that the engram is widely distributed in the brain. His experiments demonstrated that progressive lesions of a rat's brain induced similar progressive memory impairment on spatial memory tasks, but the exact location of tissue resection did not determine the extent of the deficit. Later, specific memory impairments were reported in patients with selective lesions of the hippocampus, a substructure of the medial temporal lobe (MTL). Famous studies of patients with such lesions, such as H.M. (Scoville and Milner, 1957), typically report both anterograde (deficits in forming new memories) and retrograde (deficits in retrieving old memories) amnesia specifically for episodic memories. Nonetheless, retrograde amnesia was often found to be graded: events that occurred years before the injury were still remembered, whereas more recent events were forgotten. Hence, it was proposed that

Received Jan. 18, 2013; revised Feb. 14, 2013; accepted Feb. 20, 2013.

Correspondence should be addressed to Ruud Berkers, Donders Institute for Brain, Cognition, and Behaviour, Radboud University Nijmegen, P.0. Box 9101, 6500 HB Nijmegen, The Netherlands. E-mail: r.berkers@donders.ru.nl.

DOI:10.1523/JNEUROSCI.0246-13.2013

Copyright $\odot 2013$ the authors $\quad 0270-6474 / 13 / 335435-02 \$ 15.00 / 0$ the MTL, and specifically the hippocampus, is important for the initial encoding of episodic memories, but memories later become embedded in a neocortical network (Squire and Alvarez, 1995) and therefore are not affected by MTL lesions.

The dawn of functional magnetic resonance imaging (fMRI) provided neuroscientists with a tool that appeared ideally equipped to investigate the engram throughout the brain. Overall, fMRI studies examining remote autobiographical memory retrieval demonstrated activity in a widely distributed brain network, including the hippocampus (Maguire, 2001). This unexpected hippocampal activation during remote retrieval of vivid, episodically detailed memories led to the hypothesis that hippocampal activation might not necessarily be related to the age of the memory but rather to its vividness (Gilboa et al., 2004; Winocur et al., 2010). This account suggests that the hippocampus has a persistent role in vivid retrieval of episodic autobiographical memories, whereas semantic, abstract concepts can also be retrieved independently from the MTL after consolidation. If this hypothesis is correct, the finding that features of remote memories are preserved after MTL damage can be explained if one assumes that autobiographical memories can be transformed over time, thereby losing episodic detail and becoming abstract and semanticized (Winocur et al., 2010).

Computational models conceptualize the hippocampus and the neocortex as separable systems using complementary mechanisms to learn and store information (McClelland et al., 1995). The hippocampus is thought to be a fast-learning system that rapidly stores complete events and reinstates patterns of activity in the neocortex when events are reexperienced, whereas the slow-learning neocortical system gradually stores accumulating statistical regularities and generalities across ensembles of experiences. The main nodes in this slower-learning neocortical network have been shown to include the ventromedial prefrontal cortex (vmPFC), the retrosplenial cortex (RSC), and temporal cortex (Maguire, 2001).

Recent research has started to investigate the functional integration of largescale networks during memory encoding, consolidation, and retrieval. A study by Bonnici et al. (2012) adds to these developments by using multivariate pattern analysis (MVPA) techniques to decode distributed representations of both recent and remote autobiographical memories within previously defined regions of interests. Specifically, decoding was used to establish whether certain brain regions contained memory-specific information during retrieval of remote and recent events. Individual accounts of life events were obtained through an autobiographical memory interview, of which three remote ( 10 years old) and three recent (2-3 weeks old) events were selected and matched in terms of rated vividness. 
While in the scanner, participants were cued to vividly recall these events. Subsequently, a multivariate classifier was trained to distinguish multivoxel activity patterns corresponding to either remote or recent autobiographical memories. The rationale here is that, if the retrieved memory can be decoded based on activity patterns measured in a certain brain region, then that region must represent information for at least one of the remote or recent memories. The results show that representations of both recent and remote autobiographical memories were widely distributed and could be decoded with above-chance accuracy from activity patterns in the MTL, vmPFC, RSC, and temporal cortex, respectively. Differences between remote and recent memories were observed in the vmPFC, in which decoding accuracy was significantly better for remote than for recent events. These results support a distributed model in which functional integration across hippocampus and neocortex underlie autobiographical memory retrieval. However, these results raise important questions for additional study, because it is unclear why remote and recent memories can be decoded equally well from the hippocampus and why remote memories can be decoded better from the vmPFC.

Possible explanations for the findings of Bonnici et al. (2012) can emerge by considering a narrative of a remote autobiographical event. Imagine recalling a birthday party that you had at your house approximately 10 years ago. You might describe the interior of the house, the people that were at the party, and specific interactions between the attendees. The outline of your house likely stayed the same throughout the time you lived there, but certain elements (e.g., furniture, wall colors, etc.) changed over time. Some party attendees you still encounter on a daily basis and some have since disappeared from your life and are thus more specific features of the memory. Particular events at the party have occurred multiple times at parties throughout that period (e.g., certain games and conversations), but there are also events that are specific to that one party. When retrieving this remote event, your memory will likely consist of a mixture of a few specific, episodic MTL traces, and more general, semantic neocortical traces, given that remote memories are assumed to be more fragmented and less detailed than recent memories (Rosenbaum et al., 2012). This mixed representation is additionally proposed to arise through newly added episodic features together with few remaining original event-specific details and previously extracted overlapping (semantic) features of earlier events. Statistical regularities and generalities can in turn become contextualized into a more abstract (self-referential) schema, mediated by the neocortex in general and the vmPFC in particular (van Kesteren et al., 2012). In contrast, recent memories are more likely to retain a more specific episodic quality, with fewer semantic features extracted from repeated experiences.

In the study by Bonnici et al. (2012), remote memory traces might have been influenced by the vivid retrieval during the pre-study interview 1 week earlier, and this may have biased the reconstruction of the event on the basis of stored specific episodic and general semantic features. Therefore, although the authors were careful to control for vividness, it is probable that, when participants were instructed to retrieve the event as vividly as possible, they might have (perhaps even unconsciously) inserted new details or reconstructed an experience based on semantic features extracted from multiple previous events. For example, a person who was at many parties you attended, but was not at this particular party, may be inserted into the memory.

The central point here is that, by using this particular procedure, previously semanticized traces could be reconstructed with episodic details to vividly re-experience an episode. This would explain the similar ability to decode representations of recent and remote memories from MTL activity. Furthermore, the finding that remote memories were more accurately decoded than recent memories from activity in the vmPFC suggests that this region might represent more semantic features of a memory, as mentioned above (van Kesteren et al., 2012). Such a role for the vmPFC was briefly addressed by Bonnici et al. (2012) but was countered by the statement that all memories were equally vividly re-experienced. However, when considering coexisting but dissociable contributions of brain regions to the retrieval of an autobiographical event, as described above, partial semanticization and episodic integration with preexisting knowledge structures might provide an additional interpretation of the current data.

In summary, the results from this study contribute to our understanding of autobiographical memory consolidation by demonstrating that multiple regions contain distributed autobiographical in- formation. A specific memory, in particular an autobiographical one, is suggested to consist of multiple features that are represented by different brain regions at the same time. Along with functional connectivity analyses, MVPA methods can be used to determine what information these different regions contribute and how they are functionally integrated. A next step would be to analyze feature dimensions of autobiographical memories, such as regularity across ensembles of events and vividness, and how events differing in terms of these dimensions can be decoded from different brain regions. This approach might ultimately disentangle and deconstruct the computational roles of multiple brain regions in autobiographical memory retrieval.

\section{References}

Bonnici HM, Chadwick MJ, Lutti A, Hassabis D, Weiskopf N, Maguire EA (2012) Detecting representations of recent and remote autobiographical memories in vmPFC and hippocampus. J Neurosci 32:16982-16991. CrossRef Medline

Gilboa A, Winocur G, Grady CL, Hevenor SJ, Moscovitch M (2004) Remembering our past: functional neuroanatomy of recollection of recent and very remote personal events. Cereb Cortex 14:1214-1225. CrossRef Medline

Lashley KS (1950) In search of the engram. In: Symposium of the society for experimental biology, Vol 4. New York: Cambridge UP.

Maguire EA (2001) Neuroimaging studies of autobiographical event memory. Philos Trans R Soc Lond B Biol Sci 356:1441-1451. CrossRef Medline

McClelland JL, McNaughton BL, O’Reilly RC (1995) Why there are complementary learning systems in the hippocampus and neocortex: insights from the successes and failures of connectionist models of learning and memory. Psychol Rev 102:419-457. CrossRef Medline

Rosenbaum RS, Winocur G, Binns MA, Moscovitch M (2012) Remote spatial memory in aging: all is not lost. Front Aging Neurosci 4:25. CrossRef Medline

Scoville WB, Milner B (1957) Loss of recent memory after bilateral hippocampal lesions. J Neurol Neurosurg Psychiatry 20:11-21. CrossRef

Squire LR, Alvarez P (1995) Retrograde amnesia and memory consolidation: a neurobiological perspective. Curr Opin Neurobiol 5:169-177. CrossRef Medline

van Kesteren MT, Ruiter DJ, Fernández G, Henson RN (2012) How schema and novelty augment memory formation. Trends Neurosci 35:211-219. CrossRef Medline

Winocur G, Moscovitch M, Bontempi B (2010) Memory formation and long-term retention in humans and animals: convergence towards a transformation account of hippocampalneocortical interactions. Neuropsychologia 48:2339-2356. CrossRef Medline 